[81] Randoin. Bull. Soc. Chim. Biol., 1941, 23, 437.

[82] CARY. Food and life yearbook of agric, U.S. dep. of Agric., 1939, 865, 685.

[83] Giliam et Heilbronn. Biochim. $J$., 1935, 29, 834.

[84] Van WijngaArden. Nederl. Tijdschr. V. Geneesk, 1934, 78, 2668.

[85] Sutron et Kraus. Bimo. Bull, 1936, 8, 21.

[86] Hodgson, Knott, Murer et Graves, 1958, 57, 513.

[87] Sмiтн et Powell. J, Dairy Sc., 1937, 20, 428.

[88] Meigs et Converse. Proc. Amer. Soc. Animal Production, 1933, 58 (Janv. 1934).

[89] Russel et Taylor. N. J. Agric. Exp. Sta. Bull., 1935, 592, 11.

[90] Friederichsen et With. Annales Pédiatrici, 1939, 153, 113.

[91] Shaw, Bechdel, Guerrant, Dutcher. Journ. Dairy Sc., 1937, 20, 521.

[92] Beeson. Proc. Amer. Soc. Animal. Prod., 1935, 54.

[93] Beck, Witnah, Martin. Journ. Dairy Sc., 1939, 22, 28.

[94] Cowe, Martin et Beok. Journ. Dairy Sc., 1939, 22, 457.

[95] Carson Brown. Journ. Dairy Sc., 1939, 23, 5, 351.

[96] Van WIJNGAARDEN. Dissertation Utrecht, 1935.

[97] Denel, Halliday. Hallman, Johnston, Mrller. J. Nutrit., 1941, 22, 303 .

\title{
PRODUCTION ET CONTROLE HYGIÉNIQUE DU LAIT DANS LES ÉTABLES URBAINES ET SUBURBAINES
}

\author{
par \\ ANDRÉ LOUIS \\ Docteur-Vétérinaire sanitaire \\ (Fin)

\section{CHAPITRE III \\ Hygiène du Personnel}

Le lait, provenant d'animaux sains, peut être contaminé, lors de sa récolte ou des diverses manipulations dont il est l'objet, par les personnes qui en sont chargées. Si celles-ci sont atteintes ou convalescentes de maladies contagieuses, le lait devient alors un agent d'infection.

Le lait consommé cru, a été dénoncé comme agent de transmission de la fièvre typhcïde, dès 1879, par CAMERoun relatant une épidémie qui sévit à Dublin et frappa soixante-sept personnes. Les malades avaient consommé le lait provenant d'une vacherie, dans le personnel de laquelle la fièvre typhoïde avait sévi et où leu déjections de malades avaient été jetées sur un fumier voisin ds lieu ou la traite était pratiquée. Depuis, les observations se sont multipliées, et, en 1914, Ch. Porcher pouvait établir une liste de 1.500 épidémies dont l'origine lactée a vait été démontrée d'une façon certaine.

Les observations de diphtérie transmises par le lait sont nom- 
breuses. Elles ont été recueillies surtout dans les pays anglo-saxons. Dans la plupart des foyers signalés, la vache laitière sert d'hôte intermédiaire à la contagion. L'agent pathogène est transmis à cet animal par les trayeurs infectés, probablement par suite de cette habitude malpropre et dangereuse qu'ont ces professionnels de se cracher dans les mains avant la traite pour faciliter la mulsion. De petites ulcérations dans lesquelles le germe est toujours retrouvé, se développent alors au niveau de la ma melle et en particulier sur les trayons. A son tour l'animal contamine l'homme, soit directement s'il s'agit d'un nouveau personnel préposé à la traite, soitindirectement par l'intermédiaire du lait (Deau et Todd, Ashby, GraHam et Golaz).

On a depuis longtemps observé des épidémies de scarlatine occasionnées par le lait de fermes dont le personnel était infecté (TAYLor, Power...). Au cours d'une épidémie de scarlatine à Vejle, (Danemark), en 1938, Henningsen et Ernst, ont montré le rôle du lait dans la propagation de cette infection.

L'épidémie de Vejle débuta de façon massive : 20 cas le premier jour, 40 le second. En deux semaines 118 cas de scarlatine furent observés. Le début brusque, le fait que tous les malades avaient leur habitation dans les rues avoisinant une même laiterie, firent penser à l'origine lactée. On trouva rapidement, dans une des fermes d'où venait le lait, une jeune fille trayeuse, présentant de la desquamation des mains et une décharge purulente de l'oreille, très abondante. Des eultures de la gorge et du pus otitique montrèrent la présence d'un streptocoque hémolytique B du groupe A. Lancefield, type 3 de Griffith. Dans 108 cas, le même streptocoque fut isolé. Dans 10 cas la recherche ne put être faite. L'examen des vaches qui avait fourni le lait, les montra en bonne santé, à deux reprises différentes. En somme, le lait avait été le véhicule banal du streptocoque infectant qui l'avait transporté du porteur de germes aux consommateurs sans qu'il puisse être question d'une mastite intermédiaire de la vache.

Enfin la contamination du lait par le personnel tuberculeux n'est pas à démontrer. On a retrouvé des bacilles tuberculeux du type humain dans le lait, et ceci même après pasteurisation (BOER). Ceci n'est pas surprenant, la pollution du lait par un personnel infecté pouvant se produire après la pasteurisation.

Modes de contamination du lait. - Le lait peut être contaminé de diverses façons.

Il peut être infecté à l'étable :

1. Par un convalescent de maladies contagieuses, ou un porteur chronique ou sain de bacilles typhiques ou paratyphiques. On sait 
combien un ancien typhique peut rester longtemps porteur de germes, et, s'il effectue la traite, il pourra ensemencer directement le lait au sortir des trayons. Le lait est un excellent milieu de culture, et, s'il n'est pas maintenu à basse température, quelques éléments microbiens peuvent se multiplier de telle façon que le lait devient une véritable culture en vingt-quatre heures;

20 Par l'eau servant au lavage des récipients. Dans gertaines épidémies, ce sont les déjections d'un malade, soigné à la ferme, qui étaient à l'origine de la contamination spécifique de l'eau. On lavait, dans le même bac, le linge du typhique et les ustensiles de laiterie ;

$3^{0}$ Par les personnes préposées aux soins d'un malade. C'est ce qui s'est passé pour l'épidémie de typhoïde du Kremlin-Bicêtre, décrite par DuBIEF.

Le lait peut être infecté au cours du transport, pendant son refroidissement après la pasteurisation, ou lors de sa mise en bouteilles.

Il peut être enfin infecté chez le revendeur, s'il est vendu en bidon. On y retrouve les mêmes causes de pollution qu'à l'étable : convalescents ou porteurs de germes, lavages de bidons avec de l'eau polluée, etc. Les conséquences, dans ce cas, sont parfois plus graves, parce que la pollution peut porter sur une plus grande quantité de liquide que lorsqu'il s'agit d'un producteur isolé. D'autre part, le mélange d'un lait contaminé à la ferme, avec les autres laits indemnes provenant d'autres fournisseurs constitue une source de contamination de grandes masses de lait qui peuvent devenir redoutables: Les épidémies provenant de laits, contaminés chez les revendeurs, șont nombreuses.

On se rend compte, par ce bref exposé, de la nécessité de la surveillance sanitaire du personnel utilisé à la production ou à la manipulation du lait.

Pour éviter la propagation des maladies de l'homme transmissibles par le lait, le trayeur et le personnel utilisé à la manipulation du lait devront être en bon état de santé. Toute personne atteinte ou convalescente d'une maladie contagieuse ne sera admise. ni à effectuer la traite, ni à manutentionner le lait. La circulaire du Secrétaire d'Etat à la Famille et à la Santé du 19 mai 1941 invite les préfets et les directeurs régionaux à la Famille et à la Santé "à veiller à la santé des personnes chargées de la traite et de la manipulation du lait, afin d'écarter, toutes les fois qu'il sera possible, celles qui paraitraient susceptibles de transporter des germes de maladies transmissibles. ")

L'ordonnance de Police du 15 mai 1930 sur l'hygiène du lait, 
dans son contrôle facultatif de la production laitière dans le département de la Seine, édicte dans son article 13 :

$7^{\circ}$ Le producteur ne devra employer pour l'entretien de l'étable, pour les soins à donner aux animaux, pour le service de la laiterie, la traite ou la manutention du lait, aucune personne susceptible de propager une maladie transmissible; il devra prévenir l'Inspection générale des Services techniques d'hygiène de tous les eas de maladie affectant le personnel. Ce service prescrira les mesures propres à empêcher la contamination du lait; ”

$8^{\circ}$ Les médecins du Service d'hygiène procéderont à l'examen médieal des employés au moment de leur entrée. Une fois par mois au moins, ils visiteront le personnel de l'exploitation et signaleront, par un rapport eirconstancié, toutes les observations qu'ils croiront devoir faire sur l'état de santé et de propreté du personnel, sur la salubrité des locaux d'habitation.

L'arrêté du 5 novembre 1941 , portant réglementation du contrôle hygiénique du lait dans le déparbement de l'Orne, pris en application de la circulaire de M. le Secrétaire d'Etat à la Famille et à la Santé en date du 19 mai 1941 et du 18 août 1941 constitue un modèle du genre.

Il prescrit :

Art. 2. - Les diverses manipulations du lait et de ses dérivés, tant à la production qu'au centre de traitement, pendant le transport ou sur le lieu de vente, devront être pratiquées de telle façon que ces produits ne soient pas contaminés par l'opérateur.

Les personnes employées à la production, au transport, à la centralisation ou au traitement, à la manipulation et à la vente du lait sont tenues d'observer pour elles-mêmes et leurs vêtements les règles d'une stricte propreté.

En outre, elles doivent être exempties de toutes maladies transmissibles par le lait ou les produits laitiers.

Elles ne doivent pas être porteurs de germes de ces maladies.

Dans le cas où l'une de ces personnes viendrait à être atteinte de l'une des maladies transmissibles figurant sur la liste prévue par le décret du 26 mars 1936, déclaration en serait faite à M. le Médecin inspecteur de la Santé. La personne atteinte cesserait immédiatement son travail.

Les convalescents de l'une de ces maladies ne pourront être admis ou réadmis dans leurs emplois qu'avee l'autorisation du médecin inspecteur de la Santé oú des inspecteurs-adjoints de la Santé, toutes analyses nécessaires pouvant être prescrites en vue d'établir qu'ils ne sont plus porteurs de germes.

Les personnes visées à l'alinéa 2 du présent article pourront être astreintes à présenter à l'autorité sanitaire un certificat médical de non contagiosité. Elles devront en outre, se plier à toutes les mesures de contrôle prescrite par l'autorité sanitaire, notamment en ce qui concerne la recherche des porteurs de germes de maladies transmissibles. 
Le personnel ehargé de la manipulation du lait doit done être en bon état de santé ; il doit également être très propre.

Avant de pratiquer la mulsion, le trayeur procédera à sa toilette particulière. Il revêtira un costume spécial, très propre, en toile, facilement lavable, qui ne lui servira que pour cette opération. Le plus pratique est un vêtement d'une seule pièce, dit "combinaison " ou une simple blouse laissant les bras nus. Ce vêtement sera enfermé dans un placard, en dehors des heures de traite, et non accroché dans l'étable, exposé à toutes les souillures. La coiffure sera une calotte de toile blanche, par conséquent facilement lavable et souvent lavée.

Le trayeur, avant de commencer la traite, nettoiera ses ongles, se lavera et se brossera soigneusement les mains et les bras avec de l'eau et du savon et s'essuiera avec un linge propre.

Après la mulsion de chaque animal, il se lavera à nouveau les mains. Sans cette précaution il risque de transmettre aux vaches suivantes une mammite contagieuse ignorée. Enfin ses mains devront être indemnes de toute lésion pouvant entraîner une pollution du lait (phlegmons, plaies suppuran+es, eczéma).

\section{CHAPITRE IV \\ Hygiène du Matériel}

Le matériel utilisé lors de la traite comprend : le seau à traire, le récipient commun, généralement le pot à lait, et le filtre. Ce matériel doit être imperméable et facile à nettoyer ; il ne doit pas être susceptible de donner naissance à des produits toxiques.

Les récipients métalliques, faciles à nettoyer, sont les seuls à employer. On ne peut recommander, pour la traite et pour le transport du lait, les récipients en bois, d'usage si fréquent en Hollande, en raison des difficultés de leur nettoyage. FreudenREICH rinçant avec la même quantité d'eau stérile des seaux en métal et des seaux en bois, obtint 1.105 germes par centimètre cube avec les récipients métalliques et $\mathbf{2 7 . 9 0 0}$ avec les récipients en bois.

Certains métaux, susceptibles de donner naissance à des produits toxiques, sont proscrits. L'arrêté ministériel du 28 juin 1912 relatif à la coloration, la conservation et l'emballage des denrées alimentaires et des boissons, interdit :

"De placer toutes boissons et denrées destinées à l'alimentation au contact direct du cuivre, du zinc, ou du fer galvanisé, exception faite pour les opérations de fabrication ou de conservation de produits de chocolaterie et de la confiserie ne renfermant pas de substances acides liquides, et pour les opérations de la distillerie. " 
" A u contact direct de récipients, ustensiles, appareils constitués en tout ou partie par un alliage contenant plus de $10 \%$ de plomb ou plus de $1 / 10.000^{e}$ d'arsenic."

- Au contact direct de récipients, ustensiles, appareils étamés ou soudés avec de l'étain contenant plus de $0,5 \%$ de plomb ou plús de $1 / 10.000^{\circ}$ d'arsenic. $n$

L'ordonnance de Police du 15 mai 1930 étend pour le département de la Seine cette interdiction aux récipients émaillés ou vernissés au plomb.

Les métaux qui conviennent sont : la tôle étamée à l'étain fin, l'a.luminium ou mieux ses alliages.

Le seau de traite doit avoir une ouverture réduite, orientée vers la mamelle, ce qui réduit la contamination par les poussières. Le lait recueilli dans les seaux ordinaires, largements ouverts, contient au moins deux fois plus de germes que le lait recueilli dans des récipients à étroite ouverture. Les seaux et autres récipients destinés à la récolte du lait ne doivent servir qu'à cet usage ; ils doivent présenter une surface polie et non cabossée.

Le filtre, chargé de retenir les impuretés tombées dans le lait au cours de la traite, doit être d'une propreté rigoureuse. Iait.

Los simples tamis en fil de fer ne suffisent pas pour nettoyer le

Les meilleures filtres sont les filtres d'ouate, qui consistent, en un disque d'ouate inséré entre deux plaques de métal perforés. Les disques d'ouate ne peuvent servir qu'une seule fois.

Si I'on emploie des filtres en toile, celle-ci doit être épaisse et avoir lès mailles étroites. Les filtres de toile doivent être d'abord rincés à l'eau froide après usage et ensuite seulement lavés à l'eau chaude. Après nettoyage, en hiver, lorsque I'exposition au soleil est impossible, au lieu de laisser sécher ces tissus dans une pièce habitée, au-dessus d'un poêle, il est recommandé de les conserver dans un récipient de porcelaine muni d'un couvercle et contenant un bain légèrement javellisé à raison d'une cuillerée à café par litre d'eau.

Afin que le matériel utilisé soit irréprochable, on doit immédiatement après usage, en effectuer le rinçage à l'eau froide. Nous attirons l'attention de l'usager sur la nécessité de ne pas rincer ̀̀ l'eau chaude, la chaleur provoquant la coagulation du lait qui se fixe alors plus facilement sur les parois des ustensiles. Les récipients seront ensuite soigneusement lavés et brossés à l'aide d'une solution bouillante détersive (carbonate de soude, par exemple), puis rincés à l'eau bouillie et égouttés. L'eau employée pour ce nettoyage doit être propre. Elle doit être analysée fréquemment surtout lorsqu'il s'agit d'eau de puits ou de citerne ou encore d'eau chaude obtenu à l'aide du chauffage central. On peut admettre avec 
Thinulin et Vuillaume qu'une eau propre doit avoir moins de 40 germes indologènes par litre.

La stérilisation parfaite du matériel est réalisée si l'on termine les opérations de nettoyage par un trempage prolongé ( 30 minutes) dans de l'eau javellisée à raison d'une cuillérée à café d'eau de Javel du commerce (à en viron $15-18^{\circ}$ chlorométriques) pour un litre d'eau, ce qui correspond à environ 300 milligrammes de ehlore par litre, puis par un rinçage à l'eau aseptisée à 3 milligrammes de chlore par litre. Pour réaliser ce dernier dosage il suffit de prendre une cuillérée à soupe d'eau de Javel du commerce qui ajoutée à un litre d'eau permet d'obtenir une eau-mère dont une cuillerée à café ajoutée à un litre d'eau en réalise " l'aseptisation ".

Cette précaution devrait être prise d'une façon permanente dans les établissements qui ne disposent que d'un puits ou d'une citerne d'eau potable.

Une fois le nettoyage effectué, il est indispensable de sécher le matériel ; il ne faut pas oublier que tout milieu humide est favorable au développement microbien. Plusieurs moyens sont à la disposition du producteur pour effectuer ce séchage. En période chaude, l'égouttage dans un local propre et sec ou à l'abri des poussières est indiqué ; en hiver, il convient de disposer les ustensiles autour d'une source de chaleur (poèle, fourneau...).

Avant d'utiliser les récipients, il faut en refroidir le métal extérieurement ; un rinçage à l'eau froide des récipients dans ce but est th proscrire, l'eau n'étant pas aseptique rendrait illusoires toutes les opérations précédemment pratiquées.

Si l'on effectue la traite mécanique, il est indispensablé que toutes les parties de la machine soient d'une propreté rigoureuse. Après chaque traite les tūbes trayeurs seront plongés d'abord dans l'eau froide, puis dans l'eau aseptisée, la machine continuant \& fonctionner. Il se produit ainsi un véritable lavage mécanique de toutes les parties de l'appareil. Suivant la température, les machines trayeuses seront démontées deux ou trois fois par semaine, et toutes les pièces soigneusement nettoyées.

\section{CHAPITRE V \\ Hygiène de la Traite}

L'hygiène de la traite est fonction de la propreté du local, de celle de l'animal et de celle du trayeur.

\section{LE LOCAL}

Il convient de préconiser la traite hors de l'étable dont l'air est riche en poussières et en microbes. Une salle de traite annexe 
permet d'effectuer la mulsion dans d'excellentes conditions. Sans être luxueuse, elle doit être très claire et facile à maintenir très propre. Les vaches y seront conduites par petits groupes. A défaut, ce local spécial peut être réduit à un hangar ou à un enclos situés près de l'étable, où les vaches sont rassemblées pour la mulsion. L'inconvénient de cette façon de faire est d'exposer le lait à une large contamination par les poussières atmosphériques, en cas de vent. Si, par suite du manque de locaux, la traite s'effectue à l'étable, l'enlèvement des fumiers et le pansage devront être terminés une heure au moins avant la mulsion.

Dans beaucoup d'exploitations, on distribue du fourrage aux animaux au moment de la traite pour qu'ils se tiennent tranquilles. Cette façon de procéder met en suspension dans l'étable de grandes quantités de poussières qui retombent dans le lait et le souillent , il se produit au moment de "l'affouragement" une véritable pluie de microbes (le foin contient à peu près un million de germes par gramme). Cette méthode doit être abandonnée.

En été, il faut se défendre contre les mouches qui excitent les animaux et qui viennent se désaltérer ou se noyer dans le lait, car elles apportent avec elles un grand nombre de microbes et en particulier les microbes du groupe Coli paratyphique qui sont dangereux pour le consommateur. Lorsque les fumiers sont enlevés chaque jour et déposés assez loin de l'étable, quand les fenêtres ne laissent passer que de la lumière bleue, la quantité de mouches présentes dans l'étable est toujours très faible.

Quel que soit le local de traite, il doit être muni d'un lavabo pour permettre au trayeur de se laver fréquemment les mains.

\section{L'ANIMAL.}

L'étrillage et le brossage journaliers faits en dehors de l'étable, seront complétés, avant chaque traite, par le lavage du train postérieur des animaux limité au moins á celui de la mamelle et de la partie inférieure du flanc droit. La toilette de la mamelle doit être faite à l'eau tiède et au savon, à condition de n'employer qu'un savon non caustique. Après lavage, afin d'éviter la chute de gouttes d'eau dans le seau de traite, les parties mouillées seront essuyées oigneusement avec un linge sec et propre.

Pendant la traite, la queue de l'animal est une gêne et une cause de souillure du lait. Le toupillon de l'appendice eaudal atteint le trayeur, frappe les parois du corps de l'animal et soulève toujours quelques poussières. Il est recommandé de fixer la queue au jarret gauche de l'animal par un lien ordinaire, par une courroie avec bonele ou par tout autre procédé. 


\section{LE TRAYEUR}

Ayant revêtu, au moment d'effectuer la traite, son costume spécial et s'étant lavé les mains et les bras à la brosse et au savon, le trayeur doit éviter les actes antihygiéniques qui neutralisent les précautions initiales qu'il a pu prendre. Il doit éviter, pour faire déplacer l'animal, de le pousser en appuyant la main sur la croupe ou de le caresser pour l'avertir de son approche, de déplacer, une fois les mains propres, son tabouret de traite, toujours très pollué. Un tabouret de traite à un pied en forme de champignon, fixé au corps par une courroie permet au trayeur de se déplacer sans y toucher. Ce système utilisé en Suisse et chez certains nourrisseurs de la région parisienne est à préconiser.

Le vacher ne traira pas, la tête appuyée au flane de la vache, pour éviter la chute des poils dans le lait.

Le trayeur ne crachera pas dans ses mains ou ne plongera pas périodiquement ses doigts dans le lait pour faciliter le glissement des trayons comme cela se produit encore trop souvent. C'est une pratique antihygiénique au premier chef, elle risque, en outre, si l'animal est atteint de mammite méconnue, de contaminer les voisins. C'est le plus souvent par les mains du trayeur que se transmettent les maladies de la mamelle (mammite streptococcique). Dans une étable de la Seine, ou sévissait cette pratique, 9 vaches sur 13 étaient atteintes de mammite.

Pour faciliter le glissement des doigts du trayeur, les trayons seront enduits d'un corps gras neutre, les antiseptiques étant souvent irritants. La vaseline stérile est le produit de choix.

\section{LA TRAITE}

Ces précautions prises, il faut avant la traite examiner le lait de chaque trayon pour éliminer celui qui pourrait paraitre anormal. Cet examen peut se faire très simplement en recevant le premier jet de lait de chaque quartier dans un petit gobelet ou dans une petite casserole. Si le lait d'un trayon n'apparaît pas normal avant la traite, il faut en mettre le produit à pait. De même les vaches malades seront traites à part, en dernier lieu et si possible dans un local spécial. Leur lait ne sera jamais répandu sur la litière ou sur le fumier, car il pourrait servir d'agent de contamination ; il sera placé dans un récipient séparé en vue de la stérilisation immédiate avant d'être donné aux animaux de la ferme. Il ne sera jamais livré à la consommation humaine.

Le trayeur ensuite éliminera les trois ou quatre premiers jets de ehaque trayon, parce que ces jets sont plus riches en microbes que le lait contenu dans la mamelle et qu'ils provoqueraient une 
pollution de la totalité de la traite. Dans un examen du lait au début, et en cours de traite, Freudenreich, trouve 15.563 germes par centimètre cube au début de la traite, 2.477 germes au milieu de la traite et 1.004 germes en fin de traite, de même ORLA-JENSEN trouve 16.000 germes par centimètre cube dans les premiers jets, 480 germes dans la portion moyenne et 360 dans les derniers jets. Le trayeur ne fera pas gicler ces premiers jets sur la litière, il les recevra dans un récipient spécial. La totalité de ce lait peut être soumise à l'ébullition et donnée aux animaux de la ferme.

La traite doit éliminer complètement le lait qui a été secrété par la mamelle ; elle doit être pratiquée à fond. C'est le lait de la fin de la traite qui renferme le plus de matières grasses ; en ne trayant pas à fond, on arrive donc au même résultat qu'en écrémant le lait. En particulier, si la vache allaite un veau, il faut d'abord faire têter celui-ei avant de traire : l'opération inverse, encore trop souvent pratiquée, est une fraude. En outre, s'il reste du lait, aussi peut soitil, dans la mamelle, ce résidu peut influencer la sécrétion ultérieuré ; nous nous trouvons alors en présence de laits de rétention dont le rôle est primordial dans l'étiologie des mammites. La traite pratiquée à fond constitue la meilleure prophylaxie et le meilleur traitement de la mammite.

Le lait trait sera, vache par vache, versé immédiatement dans un récipient commun (pot à lait) sur lequel est disposé le f.ltre.

Presque toujours le trayeur attend que son seau à traire soit plein pour le vider ; cette méthode est à condamner, car souvent il interrompt la traite d'un animal, le seau étant plein, et pour le vider, il le saisit d'une main par l'anse, de l'autre par le fond qui repose sur la litière, puis il reprend la traite de son animal, sans se laver les mains, ne songeant pas à quel point il a pu les polluer par cette mancuvre.

Après la filtration le lait sera couvert pour éviter toute pollution par les poussières ou par les mouches. Les bidons ne doivent pas être fermés avec le couvercle, mais avec une mousseline double ou une toile propre, afin d'en permettre l'aération et d'en faciliter le refroidissement.

Pour pratiquer la traite correctement, le trayeur effectuera ses opérations dans l'ordre suivant:

Il fixe la queue au jarret de la première vache ;

Il fait la toilette de la mamelle ;

Il se lave les mains ;

Il effectue la traite de la première vache ;

Il verse le contenu du seau de traite dans le réeipient commun ;

Il passe à la vache suivante sur laquelle il répète dans le même ordre les mêmes opérations. 


\section{CHAPITRE VI}

\section{Soins à donner au lait après la traite Son contrôle hygiénique avant la vente}

Le lait qui vient d'être trait possède une température voisine de celle du corps de la vache, e'est-à-dire $37^{\circ}$. A cette température, les rares microbes qu'il peut contenir pulluleraient avec facilité si l'on n'intervenait pas par un refroidissement immédiat et énergique et s'il n'existait un phénomène inhibant la pullulation des germes pendant les trois ou quatre heures qui suivent la traite. Ce phénomène constitue la "phase bactéricide " au cours de laquelle le nombre de microbes n'augmente pas. Il faut done profiter de cette circonstance heureuse pour abaisser suffisamment la température du lait et le protéger avant la disparition de la phase bactéricide.

Plus le refroidissement est rapide et plus il est intense, mieux le lait se conserve. Le froid ne tue pas les microbes, mais il les paralyse et il les empêche de proliférer.

Aussitôt après la traite, le lait doit done être éloigné de l'étable, à cause de la température qui y règne, et refroidi. Les pots seront plongés, au fur et à mesure de leur remplissage, dans des bassins contenant de l'eau aussi fraîche que possible. Cette eau sera courante ou à défaut, souvent renouvelée. Ce refroidissement se pratiquera aussi bien en été qu'en hiver. Dans les exploitations importantes on pourra utiliser de préférence des réfrigérants à glace ou de véritables appareils frigorifiques. Ils nous permettent de nous rapprocher le plus possible de la température idéale de conservation du lait qui est de $4^{\circ} \mathrm{C}$. On ne dépassera jamais la température de $12^{\circ} \mathrm{C}$. qui est pour le lait une véritable température critique.

Il est indiqué de ne pas mélanger la traite du matin et celle du soir. Si l'une des traites est altérée, l'autre s'altérera par le mélange: on a toujours en effet observé qu'en mélangeant le lait bon avec le mauvais, le mauvais ne s'améliore pas et le bon se gâte.

Une fois le lait refroidi, les pots seront refermés avec leur couvercle et conservés au froid, ou bien, ce qui est préférable, le lait sera mis en bouteille.

"L'embouteillage du lait constitue la dernière étape d'une production rationnelle et sa mise en bouteille dès l'etable est une condition essentielle de sa qualité hygiénique (Threvlin). n. Cette opération réduit au minimum les manipulations et les transvasements dont le lait est l'objet, elle en diminue done les chances do pollution. 
La vente du lait "à la mesure » constituant une pratique condamnable au point de vue hygiénique, on devra prévoir des bouteilles de trois capacités : litre, demi-litre, quart de litre.

La bouteille sera en verre, d'une forme présentant le maximum de facilités pour le nettoyage et une bonne stabilité : bouteille à fond plat, à angle arrondi, à corps cylindrique et à épaulement ogival.

Le verre sera transparent, ce qui permet de contrôler aisément la propreté du récipient.

Le système de bouchage qui doit être adopté est indiscutablement le eapsulage au moyen de capsules d'aluminium ; il protège parfaitement le lait de toutes souillures.

Le nettoyage des bouteilles exige d'abord un lavage à l'eau froide, puis un trempage dans l'eau carbonatée chande et le goupillonnage, ensuite un rinçage à l'eau potable ou à défaut à l'eau aseptisée à 3 milligrammes de chlore par litre, enfin l'égouttage à l'abri des poussières.

L'efficacité du lavage est surtout une question de durée effective des opérations et de température des bains de lavage ; il doit nous garantir des bouteilles bactériologiquement propres.

Un examen au laboratoire de bouteille prêtes pour le remplissage, bouchées avec un bouchon de coton stérile, nous renseignera sur l'efficacité du lavage. Cet examen eomporte la numération des colonies microbiennes sur plaque de gélose et la numération des germes indologènes.

Nous apprécierons, a vec Thieulin et Vuillaume, la propreté des récipients, selon le tableau suivant :

Par centimètre cube :

Moins de 2 germes indologènes et de 500 colonies

Entre 2 et 20 germes indologènesou

500 et 2.500 colonies .......... flacon malpropre

Au-dessus de 20 germes indologènes

ou de 2.500 colonies ........... flacon très malpropre

A ce dernier stade de la production, au moment où le lait va être mis en vente, un nouveau contrôle hygiénique nous renseignera utilement sur l'efficacité des mesures prises, et sur la pollution du lait lors de la traite ou des diverses manipulations qui ont suivi cette opération.

Ce dernier contrôle comporte un examen sur place et un examen an laboratoire. 


\section{a) Examen sur place}

Cet examen comporte une épreuve effectuée au lieu de distribution ou de vente : la lacto-filtration.

\section{Lacto-filtration.}

Cette épreuve a pour but de mettre en évidence les impuretés macroscopiques du lait, d'origine extra-mammaire, en les retenant par filtration sur une rondelle d'ouate. On peut ainsi déterminer son degré de propreté, la nature des souillures et reconnaître les laits malpropres qui n'auraient pas été décelés par un simple examen des caractères physiques.

Les résultats, pour être comparables entre eux, doivent toujours être obtenus avec le même volume de lait $\left(500 \mathrm{~cm}^{3}\right)$ et à travers la même surface de rondelle.

Le lacto-filtreur est une bouteille sans fond, d'une capacité dé trois quarts de litre environ, dont le goulot peut être fermé à l'aide d'une rondelle d'ouate de 2,5 à 3 centimètres de diamètre, maintenue en place par une toile métallique inattaquable et une agrafe.

Le lacto-filtreur étant placé l'ouverture en haut, on y introduit $500 \mathrm{~cm}^{3}$ de lait. Il est recommandé d'opérer sur les fonds de récipient, ou, par suite de leur poids, toutes les impuretés tombent. Le liquide filtre à travers la rondelle qui retient les impuretés solides. Lorsque la filtration est terminée, on dégage la rondelle d'ouate et on la fixe sur une fiche de référence pour examen comparatif. Une échelle de cotation de 0 à 5 donne toute la gamme des laits malpropres.

Cette épreuve frappe beaucoup le producteur qui se rend compte, par lui-même, de la malpropreté de son lait. Une teneur microbienne élevée ne parle pas à son esprit ; une rondelle de filtration remplie de fumier le persuade bien plus facilement de sa négligence. Les améliorations, transcrites sur le graphique ci-contre (fig. 2), obtenues, dans le département de la Seine, par cette simple épreuve, sont concluantes.

Cet examen nous renseigne sur la façon dont le producteur a effectué la filtration de son lait. Cette opération, quil est indispensable, retient toutes les impuretés (débris épithéliaux, poils, parcelles de paille, de fourrages divers, d'excréments) qui ont pu tomber dans le lait pendant la traite et évite que ces matières ne macèrent dans cet aliment jusqu'au moment de sa consommation. Elle ne dispense toutefois pas d'éviter que, pendant la traite, ces impuretés. ne tombent dans le lait. Les bactéries qui accompagnent les particules excrémentitielles tombées dans le lait, ont souvent abandonné leur substratum pour envahir la masse liquide et ne demandent qu'une température favorable pour pulluler. La meilleure 
filtration ne pourra pas les retenir. C'est pourquoi il ne faut pas que le trayeur, comptant sur l'efficacité de son filtre, ne prenne aucun soin pendant la traite pour éviter la pollution de son lait. Nous avons trop souvent constaté que les producteurs avaient recours à cette solution de paresse. De même que, sous prétexte qu'il va être pasteurisé par la suite, un lait ne doit pas être recueilli au

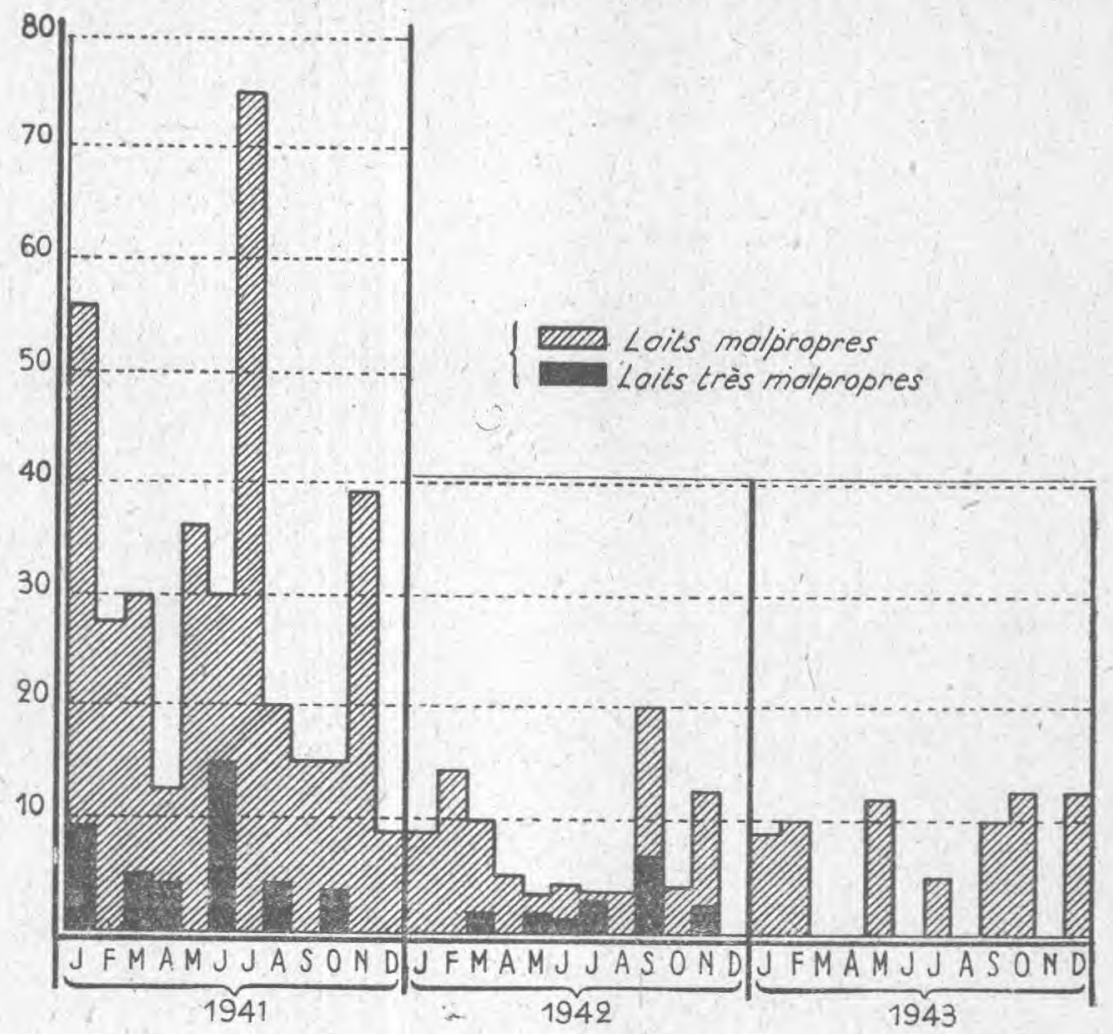

Fig. 2. - Lacto-filtrations

(Rapport du Service Sanitaire Vétérinaire de la Seine)

mépris de toutes règles d'hygiène, de même, sous prétexte qu'il va être filtré, un lait ne doit pas être récolté sans souci des saletés et des excréments qui peuvent y tomber. L'emploi du filtre ne dispense pas d'observer les règles d'une traite hygiénique, il ne constitue qu'un moyen complémentaire d'obtenir un lait propre.

La lacto-filtration ne nous renseigne que sur la propreté macroscopique du lait, il faut avoir recours au laboratoire pour être renseigné sur son degré de pollution au cours de la traite et des diverses manipulations $q u$ 'il a subies. 


\section{b) Examen au laboratoire}

En vue de cet examen, des prélèvements seront effectués au lieu de distribution ou de vente.

Si le lait est livré en bouteille, une de celles-ci sera envoyée au laboratoire ; s'il est livré en pot, un prélèvement aseptique sera fait sur ces pots. Il sera effectué à l'aide d'une grande pipette de 50 à $125 \mathrm{~cm}^{3}$ et d'un flacon stérilisés. En prineipe, l'analyse au laboratoire nécessite le prélèvement d'un échantillon de lait de $125 \mathrm{~cm}^{3}$ dans un flacon à fermeture hermétique (fermeture canette de préférence). La pipette n'est sortie de son enveloppe de papier qu'au moment du prélèvement; le flacon n'est ouvert qu'au moment d'y introduire le lait prélevê à la pipette et fermé aussitôt après, en ayant soin de ne pas toucher la partie inférieure du bouchon avec les doigts.

La pollution du lait nous sera révélée par l'épreuve de la réductase microbienne et par la numération des germes indologènes.

La numération des colonies microbiennes sur plaque de gélose qui nous fournit de précieux renseignements pour les laits pasteurrisés est sans valeur pour le lait cru où seule la qualité des germes compte.

\section{Epreuve de la réductase microbienne.}

Les microbes du lai secrétent une diastase (réductase microbienne) capable de rédu.re certaines substances. On choisit une substance colorée telle que le bleu de méthylène, donnant, par réduction, un leuco dérivé incolore.

La rapidité de décoloration du bleu de méthylène est surtout fonction de quantité de mierobes présents ; cependant leur qualité intervient (ainsi le streptocoque de la mammite contagieuse ne décolore pas le bleu de méthylène) mais, dans l'ensemble, la mesure du temps de décoloration peut permettre de se faire une idée du degré de contamination du lait.

La décoloration a lieu en moins

d'une heure ............ lait fortement contaminé

La décoloration a lieu entre 1 et

3 heures .............. lait passable (qualité courante)

La décoloration a lieu au-dessus

de 3 heures ........... lait de bonne qualité

Numération des germes indologènes.

L'existence dans le lait d'Escherichia Coli (colibacille, bacille du côlon) est l'indice de souillures importantes. Si sa présence à un faible taux peut être considéré comme habituelle dans les laits 
crus, sa présence à un taux élevé indique une pollution de ces laits.

La numération de ces germes indologènes nous permet d'appréeier, en nous référant au tableau suivant de THIEULIN et VuILLAUME, l'importance des contaminations du lait pendant et après la traite.

Pour le lait oru (par centimètre cube).

\section{Moins de 10 germes indolo-}

gènes ............... lait de bonne qualité

Entre 10 et 100 germes .... lait de qualité courante, Entre 100 et 1.000 germes ... lait de qualité inférieure Plus de 1.000 germes ....... lait très souillé

Le lait sera conservé au froid, en glacière, jusqu'à sa distribution. Le cycle de la production hygiénique de notre lait est terminé, il arrive propre et sain au consommateur. Il importe de donner à ce dernier quelques conseils pour l'utilisation de son lait, afin que, par des manceuvres maladroites, il ne réduise à néant toutes les précautions prises pour lui donner un produit de qualité. Nous préconisons l'affichage, dans les locaux de distribution et de vente, de ces quelques conseils destinés au consommateur :

\section{Avis important}

Tous les récipients servant au transport, à la cuisson ou d̀ la conservation du lait, doivent être d'une propreté rigoureuse el munis d'un couvercle. Lavez-les soigneusement après usage et égouttez-les.

N'ajoutez jamais à votre lait du bicarbonate de soude car s'il empêche quelquefois votre lait de tourner, il favorise toujours sa putréfaction.

Malgré toutes les précautions prises, il est recommandé de soumettre votre lait à l'ébullition. Celle-ci aura l'énorme avantage de rendre le lait de vache plus digestible pour votre nourrisson.

Conservez, à tous moments, votre lait dans l'endroit le plus frais dont vous pouvez disposer et à l'abri de toute souillure.

\section{CONCLUSION}

La création et la multiplication d'étables urbaines et suburbaines destinées à fournir à nos enfants un lait de qualité; pur propre et sain, est une nécessité. Elle correspond à un double point de vue, économique et hygiénique.

Le contrôle hygiénique de leur production ne peut être confié qu'à un vétérinaire qui seul possède les connaissances nécessaires pour en suivre la marche, de l'étable au consommateur. Les Services Vétérinaires sanitaires municipaux, disposant d'un personnel 
qualifié et de laboratoires, sont tout indiqués pour assurer ce contrôle.

Au point de vue économique, nous savons que les ravages causés par la mammite et notamment par la mammite streptococcique, se chiffrent chaque année par milliards. DrIEUX estime, en effet, que la quantité de lait perdue pour cause de mammite peut s'élever pour une année en France, à environ 970 millions de litres, sans préjudice des graves inconvénients que présentent pour l'industrie laitière les variations de la qualité du lait provenant des vaches en puissance d'infection. Dès 1941, le service vétérinaire sanitairè de la Seine, entreprenait, dans ee département, la lutte systématique eontre ce fléau. Si, en $1942,7,3 \%$ des vaches étaient encore atteintes de mammite streptococoique, ce chiffre tombait à $2,4 \%$ en 1943, tandis que dans le même temps le chiffre de mammites banales passait de $3 \%$ en 1942 à $1,5 \%$ en 1943 .

Les conseils d'hygiène, la mise à l'index des femelles contagieuses, souvent éliminées spontanément, la prise en main des malades ou suspectes par le propriétaire-jusque là non tenu par le personnel au courant de l'état de son cheptel, ont abouti à ce résultat.

A. Thuillier a essayé d'estimer approximativement l'économie réalisée par l'application de ces mesures en 1943 par rapport à l'année 1942. Il la chiffre pour les 2.709 vaches des nourrisseurs de la Seine à 1.500.000 francs. "Que faudrait-il penser, ajoute-t-il, des bénéfices que vaudrait l'application de cette méthode de contrôle aux 4 à 5 millions de vaches laitières de la France (1) chez lesquelles les mammites sont bien plus fréquentes qu'à Paris où l'état sanitaire des vaches est l'objet de la plus grande attention. " Ils seraient de l'ordre de plusieurs milliards.

Les étables urbaines et suburbaines concourront avec succès à la diffusion des méthodes de détection des mammites; elles seront autant de "stations d'essai ", où, à proximité de leur clientèle, les praticiens pourront puiser tous enseignements utiles ; elles constitueront, en outre, des "étables modèles " pour la production hygiénique du lait et représenteront pour l'ensemble de la production, le but vers lequel il faut tendre.

La pasteurisation effective des laits anonymes étant reconnue comme une mesure de salut public, il est indispensable que cette opération ne devienne pas le palliatif d'une production défectueuse. En tout état de causé, l'amélioration de la qualité hygiénique de cette production est une condition primordiale du progrès cherché : nos étables urbaines et suburbaines, fournissant sur place un lait

(1) Bulletin de statistique agrieole : le nombre des vaches laitières ne travaillant pas était de 4.872.967, en mai 1943 . 
satisfaisant, sont destinées, en dehors de leur office immédiat, à servir d'exemples tout en constituant la meilleure des propagandes en faveur de cet aliment exceptionnel qu'est le lait.

Nous insistons sur l'importance des résultats à obtenir en rappelant les déclarations du Professeur Ch. Porcher au Congrès International de Londres, en 1914 : "Les hécatombes d'enfants qui surviennent au temps chaud, les épidémies de fièvre typhoïde d'origine lactée, beaucoup plus nombreuses et meurtrières qu'on le croit, sont en relation étroite avec une large pollution du lait au moment de la traite. Les statistiques de tous les pays montrent que si la diarrhée infantile décime les enfants au-dessous de deux ans c'est le lait sale qu'il faut partout considérer comme la cause primordiale de cette mortalité. "

Dans notre travail, nous avons posé les diverses conditions qui doivent permettre de produire un lait présentant des garantie hygiéniques certaines et nous avons insisté sur les soins élémentaires qui, a eux seuls, constituent un important facteur de progrès.

Aussi, ferons-nous nôtres, les conclusions de H. Mantel dans son étude sur les "Conditions Hygiéniques de la Production du Lait » :

"PAsteur a sauvé des milliers de vies humaines en apprenant aux chirurgiens à se laver proprement les mains et à aseptiser les instruments avant de s'en servir ; de même il faut aujourd'hui apprendre anx vachers a traire proprement les vaches. C'est encore le meilleur moyen d'assurer aux nourrissons un lait salubre et de sauver ainsi nombre d'existences. "

\section{BIBLIOGRAPHIE}

P. AUFFret. L'amélioration de la production hygiénique du lait dans le département du Finistère. Thèse Doct. Vét., Paris -Alfort, 1940.

L. Babonneix. Les régimes chez l'enfant. Masson et Cie, édit., Paris, 1936.

F. Beaufrìre, R. Gely et G. Thinulin. L'inspection sanitaire du lait à l'étable dans lé département de la Seine. Bul. Acad. Vét., France, $1942, \mathrm{XV}, \mathrm{n}^{\circ} 1$.

G. Bourgeors. Le eontrôle hygiénique du lait à Dijon. Le Lait, 1938, XVIII, 648.

A. Chevisch. Sur l'hygiène et le contrôle du lait. L'approvisionnement en lait. Le Lait, 1927 , VII, 787.

G. Cordier (Mne) et L. C. BRumpt. Hémo et lacto-agglutinations rapides appliquées au diagnostic et à la prophylaxie des brucelloses, Bul. Acad. Méd., 1943, CXXVII, 10.

G. Daspet. Recherehe de I'infection brucellique du lait. Thèse-Doct. Vét., Paris-Alfort, 1945.

DhснамвRE. La vache laitière. Libr. agricole "Maison Rustique "z édit,, 26 rue Jacob, Paris, 1926. 
R. Détrez. L'embouteillage du lait en France (deux brochures de 20 et 23 pages). P. Chanove, imprimeur, Courbevoie, décembre 1941 et mai 1942, Paris, 16, rue Guynemer $\left(6^{e}\right)$; Lyon-Villeurbanne, 5, rue AntoninPerrin ; Le Lait, 1943, XIII, 149.

H. Drieux. Les mammites et leurs conséquences du point de vue de l'industrie laitière. C. R. Trav. Journée du lait, Inst. Nat. Agro., Paris, 1943, 48.

R. Gruy et G. Thinolin. L'inspection sanitaire du lait à l'étable en vuede la prophylaxie et du traitement des mammites, et, en particulier, de la mammite streptococcique. Bul. Acad. Vét. France, 1943, XVI, no 11.

G. J. Huoker et P. A. Pansen. Valeur relative des examens dans la mammite chronique des bovidés. Le Lait, 1934, XIV, 226 et 337.

JAviLIIER. Le lait et le ravitaillement vitaminique de l'homme adulte. C. R. Acad. Agric., 1940, n० 11, 396.

A. M. Leroy. Ce que doit savoir un bon contrôleur laitier et beurrier. Le Lait, $1925, \mathrm{~V}, 126$ et 278.

R. Lotr. Les malpropretés visibles du lait. Leur examen microcsopique. Le Lait, $1930, \mathrm{X}, 555$ ot 812 .

H. Martel. Conditions hygiéniques de la production du lait. Hygiène de l'étable. Hygiène de la peau. Hygiène de la traite, Rev. Soc. Scient, Hyg. Alim., 1909, VII, 1 .

G. Mignolet. Les bases légales du contrôle hygiénique du lait. Thèse Doct. Vét., Paris-Alfort, 1940.

A. Monvorsin. Le lait et les produits dérivés. Vigot frères, édit., Paris, 1925.

G. et R. Moussu. Traité des maladies du gros bétail. Asselin et Houzeau, édit., Paris, 1922.

L. Pannisset. Traité des maladies infectieuses des animaux domestiques. Vigot frères, édit., Paris, 1938.

C. H. Pérard, Rapport sur les opérations du Service Vétérinaire de Paris et du département de la Seine, 1943 et 1944. Préfecture de Police, Paris.

A. Pzerndoux. Manuel de législation sanitaire vétérinaire. Mistral, édit., Cavaillon, 1940.

Ch. Porcher. L'infection latente de la mamelle et ses réveils. Les moyens de la dépister. Le Lait, 1932, XII, 1932, 257, 431, 793 et 914; 1933, XIII, 734.

A. Roohatx et A. Tapernoux, Le lait et ses dérivés. Vigot frères, édit., Paris, 1942.

A. Tapernoux. La production d'un lait propre et sain à la ferme. Le Lait, 1937, XVII, 241.

G. Threulin. L'hygiène du lait en France. Bul. Off. Inter. Hyg. Pub., 1936, XXVIII, $\mathrm{n}^{\circ} 1$.

G. Thinumin. Méthode de contrôle hygiénique du lait. Le Lait, 1942, XXII, 8.

G. Threvun. Organisation de l'hygiène du lait en France. Bull. Acad. Méd., 1942, CXXVI, 264.

G. Thisulin et R. Vuillaume. Eléments pratiques d'analyse et d'inspection du lait. Le Lait, 16,rue Spontini (XVI $\left.{ }^{e}\right)$, édit., Paris, 1942.

A. ThurLlingr. Le eontrôle hygiénique du lait à l'étable dans le département de la Seine. Thèse Doct. Vét., Paris-Alfort, 1945. 
H. Vallée et L. Panisset. Les tuberculoses animales. Oetave Doin et Fils, édit., Paris, 1920.

J. VERGE et G. ThIEULIN. L'utilisation des laits tuberculeux. Le Lait, 1937 XVII, 348 .

R. Vulllaume. Etat actuel de nos connaissances sur les vitamines du groupe B. Rec. Méd. Vét., Alfort, 1943, CXIX, 177, 1944, CXX, 17.

\section{BIBLIOGRAPHIE ANALYTIQUE}

\section{LES LIVRES}

\section{Dornic (P.) et Chollet (A.).- Lait, Beurre et Dérivés. II. Tech-} nique laitière, beurrière et caséinière. 1 vol. broché 320 p. 96 fig., nombreux tabl. J. B. Baillière et fils, 19, rue Hautefeuille, Paris, éditeurs, 1946. Prix : $100 \mathrm{fr}$.

La seconde édition de cet ouvrage bien connu des techniciens de l'industrie laitière est en tous points digne de ses auteurs. Ce volume constitue la base des connaissances relatives à la technique laitière, beurrière et caséinière.

Après un exposé rapide des considérations de base, le chapitre I concerne la récolte, le transport et le paiement du lait (traite, manuelle et mécanique; soins à donner au lait, ramassage et paiement).

Le chapitre II est consacré à l'important problème de l'eau en industrie laitière (quantité nécessaire, puits et sources, purification des eaux destinées au lavage du beurre ; évacuation et épuration des eaux résiduaires).

Le chapitre III ótudie le froid artificiel (machines frigorifiques, isolation, congélation du beurre).

Le chapitre IV traite de la vente du lait en nature et passe en revue les conditions générales de l'entreprise, puis tous les détails pratiques qui la régissent; il est ensuite parlé des manipulations, de la conservation, de la transformation en lait concentré ou sec et de l'obtention des laits fermentés.

Le beurre est l'objet du long chapitre suivant, et l'on trouve dans l'exposé aussi complet que minutieux tout ce qui concerne l'industrie beurrière (construction et aménagement des ateliers, opérations successives, points particuliers d'ordre commercial, altération du beurre et conservation, tenue des livres, rendement, etc.).

Le chapitre VI est réservé aux laits écrémés, et, tout naturellement, l'industrie easéinière fait l'objet d'importants développements (différentes fabrications, utilisations du sérum, etc.).

Enfin, le dernier chapitre rappelle les dispositions législatives essentielles.

Il est inutile de souligner ici l'intérêt et la valeur de cette seconde édition ; nous espérons avoir bientôt le grand plaisir de présenter le tome I qui sera consacré à la seience laitière.

G. Thieutin.

Orla-Jensen (S.), Olsen (E.) et Geill ( T.). - La sénilité et la flore intestinale. Nouvel examen de la théorie de Metchnikoff. Académie Royale danoise des Sciences. Etudes biologiques, 